Journal of Universal Mathematics

Vol.2 No.2 PP.144-153 (2019)

ISSN-2618-5660

\title{
TEMPORAL INTUITIONISTIC FUZZY TOPOLOGY IN CHANG'S SENSE
}

\author{
FATIH KUTLU
}

\begin{abstract}
In this paper, the concepts of temporal and overall intuitionistic fuzzy topology in Chang sense is introduced and investigated some properties of these concepts. Furthermore, we give fundamental definitions related to this topology as temporal and overall aspects. We also examine the relationship between this topology and the temporal and overall intuitionistic fuzzy topology in Šstak's sense.
\end{abstract}

\section{INTRODUCTION}

After fuzzy set was introduced by Zadeh in 1965, various concepts defined by the classical set are also defined by this new approach and have found many different field applications, mainly engineering. In addition, a number of generalizations of the fuzzy set have been defined to handle real-world problems more effectively. One of these generalizations is intuitionistic fuzzy set (IFS for short) which was introduced by Atanassov in 1983. In this concept of intuitionistic fuzzy set, each element has two degrees named degree of membership and degree of non-membership to IFS respectively [8]. Furthermore, in this theory, it is possible to handle real world problems more realistic with hesitation degree which is the calculated by the former two degree as well as in the positive and negative aspects of an event. There are basically two different approaches to the concept of topology generated by fuzzy sets. The first approach is the definition of fuzzy topology, which is the generalization of classical topology definition and defined by Chang in 1968 [2]. This approach was generalized to intuitionistic fuzzy set theory by Coker in 1997.

Fuzzifying of topology concept was made by Šostak in 1985 [1]. In his definition, openness and closeness of fuzzy sets are graded among 0 and 1. In 1996, Çoker and Demirci [4] introduced the concept of intuitionistic fuzzy set in Šstak's sense and gave fundamental definitions and properties of it. A widely literature on both theories is presented in the references. Moreover, it can be seen that from references that there is a categorical equivalence between these two theories.

Temporal intuitionistic fuzzy set, another approach in which temporal variables also participated in calculating the membership and non-membership degrees, was defined by Atanassov in 1991 [9]. This is one of the most important extensions

2000 Mathematics Subject Classification. 47S4.

Key words and phrases. Temporal intuitionistic fuzzy sets, Temporal intuitionistic fuzzy topology, Continuity, Interior, Closure. 
of IFS. In recent years, Šostak's mean temporal intuitionistic fuzzy topology was defined by Kutlu and Bilgin [6]. Also, the other fundamental concepts of Sostak's mean temporal intuitionistic fuzzy topology defined by the author in $[5,6,7]$.

This study is organized as follows: In section 2, we give basic definitions of intuitionistic fuzzy sets and temporal intuitionistic fuzzy sets. In section 3, we introduce the concept of temporal intuitionistic fuzzy topology in Chang's sense and give some fundamental properties of it. Then overall intuitionistic fuzzy topology in Chang's sense is defined. Also it is examined that the relationship between this topology and the temporal and overall intuitionistic fuzzy topology in Šostak's sense.

\section{Preliminaries}

In this section, it is provided the basic definitions and theorems that we will use in the next section. Firstly, The definition of intuitionistic fuzzy set will be given.

Definition 2.1 (8). An intuitionistic fuzzy set in a non-empty set $X$ given by a set of ordered triples $A=\left\{\left(x, \mu_{A}(x), \eta_{A}(x)\right): x \in X\right\}$ where $\mu_{A}(x): X \rightarrow I, \eta_{A}(x)$ : $X \rightarrow I$ and $I=[0,1]$, are functions such that $0 \leq \mu(x)+\eta(x) \leq 1$ for all $x \in X$. For $x \in X, \mu_{A}(x)$ and $\eta_{A}(x)$ represent the degree of membership and degree of non-membership of $x$ to $A$ respectively. For each $x \in X$; intuitionistic fuzzy index of $x$ in $A$ can be defined as follows $\pi_{A}(x)=1-\mu_{A}(x)-\eta_{A}(x) . \pi_{A}$ is the called degree of hesitation or indeterminacy.

By $\operatorname{IFS}(X)$, we denote to the set of all intuitionistic fuzzy sets.

Definition $2.2(8)$. Let $A, B \in I F S(X)$. Then,

(i) $A \subseteq B \Leftrightarrow \mu_{A}(x) \leq \mu_{B}(x)$ and $\eta_{A}(x) \geq \eta_{B}(x)$ for $\forall x \in X$,

(ii) $A=B \Leftrightarrow A \subseteq B$ and $B \subseteq A$,

(iii) $A^{c}=\left\{\left(x, \eta_{A}(x), \mu_{A}(x)\right): x \in X\right\}$,

(iv) $\bigcap A_{i}=\left\{\left(x, \wedge \mu_{A_{i}}(x), \vee \eta_{A_{i}}(x)\right): x \in X\right\}$,

(v) $\bigcup A_{i}=\left\{\left(x, \vee \mu_{A_{i}}(x), \wedge \eta_{A_{i}}(x)\right): x \in X\right\}$,

(vi) $\tilde{0}=\{(x, 0,1): x \in X\}$ and $\tilde{1}=\{(x, 1,0): x \in X\}$.

Definition $2.3(3,8)$. . Let $a$ and $b$ be two real numbers in $[0,1]$ satisfying the inequality $a+b \leq 1$. Then, the pair $\langle a, b\rangle$ is called an intuitionistic fuzzy pair. Let $\left\langle a_{1}, b_{1}\right\rangle$ and $\left\langle a_{2}, b_{2}\right\rangle$ be two intuitionistic fuzzy pair (briefly IF-pair). Then define

(i) $\left\langle a_{1}, b_{1}\right\rangle \leq\left\langle a_{2}, b_{2}\right\rangle \Leftrightarrow a_{1} \leq a_{2}$ and $b_{1} \geq b_{2}$,

(ii) $\left\langle a_{1}, b_{1}\right\rangle=\left\langle a_{2}, b_{2}\right\rangle \Leftrightarrow a_{1}=a_{2}$ and $b_{1}=b_{2}$,

(iii) If $\left\{\left\langle a_{i}, b_{i}\right\rangle ; i \in J\right\}$ is a family of intuitionistic fuzzy pairs, then $\vee\left\langle a_{i}, b_{i}\right\rangle=\left\langle\vee a_{i}, \wedge b_{i}\right\rangle$ and $\wedge\left\langle a_{i}, b_{i}\right\rangle=\left\langle\wedge a_{i}, \vee b_{i}\right\rangle$,

(iv) The complement of $\langle a, b\rangle$ is defined by $\overline{\langle a, b\rangle}=\langle b, a\rangle$,

(v) $1^{\sim}=\langle 1,0\rangle$ and $0^{\sim}=\langle 0,1\rangle$. .

Definition 2.4 (3). An intuitionistic fuzzy topology in Šostak's sense (briefly, SIFS) on a nonempty set $X$ is an IFF $\tau$ defined with $\tau(A)=\left(\mu_{\tau}(A), \eta_{\tau}(A)\right)$ on $X$ satisfying the following axioms:

(T1) $\tau(\tilde{0})=1^{\sim}$ and $\tau(\tilde{1})=1^{\sim}$,

(T2) $\tau\left(A_{1} \cap A_{2}\right) \geq \tau\left(A_{1}\right) \wedge \tau\left(A_{2}\right)$ for any $A_{1}, A_{2} \in \operatorname{IFS}(X)$,

(T3) $\tau\left(\bigcup A_{i}\right) \geq i \in J \wedge\left(\tau\left(A_{i}\right)\right)$ for any $\left\{A_{i} ; i \in J\right\} \subseteq I F S(X)$.

The pair $(X, \tau)$ is called an intuitionistic fuzzy topological space in Šstak sense. For any $A \in I F S(X)$, the number $\mu_{\tau}(A)$ is called the openness degree of $A$, while $\eta_{\tau}(A)$ is called non-openness degree of $A$. 
Definition 2.5 (4). An intuitionistic fuzzy topology in Chang's sense (briefly, CTIFS) on a non-empty set $X$ is a family $\tau_{t}$ of TIFSs satisfying the following axioms:

I. $\tilde{0} \in \tau$ and $\tilde{1} \in \tau$,

II. $A_{1} \cap A_{2} \in \tau$ for each $A_{1}, A_{2} \in \tau$,

III. $\bigcup_{i \in I} A_{i} \in \tau$ For any arbitrary family $\left\{A_{i} ; i \in I\right\} \in \tau$,

Definition 2.6 (9). . Let $E$ be an universe and $\mathrm{T}$ be a non-empty time-moment set. We call the elements of $T$ "time moments". Based on the definition of IFS, a temporal intuitionistic fuzzy set (breifly TIFS) $A$ is defined as the following: $A(T)=\left\{\left(x, \mu_{A}(x, t), \eta_{A}(x, t)\right):(x, t) \in E \times T\right\}$ where:

(a) $A \subseteq E$ is a fixed set

(b) $\mu_{A}(x, t)+\eta_{A}(x, t) \leq 1$ for every $(x, t) \in E \times T$

(c) $\mu_{A}(x, t)$ and $\eta_{A}(x, t)$ are the degrees of membership and non-membership, respectively, of the element $x \in E$ at the time moment $t \in T$

By $T I F S^{(X, T)}$, we denote to the set of all TIFSs over nonempty set $X$ and timemoment set $T$. For brevity, we write $A$ instead of $A(T)$. The hesitation degree of a TIFS is defined $\operatorname{as} \pi_{A}(x, t)=1-\mu_{A}(x, t)-\eta_{A}(x, t)$. Obviously, every ordinary IFS can be regarded as TIFS for which $\mathrm{T}$ is a singleton set. All operations and operators on IFS can be defined for TIFSs.

Definition 2.7. Let $A\left(T^{\prime}\right)=\left\{\left(x, \mu_{A}(x, t), \eta_{A}(x, t)\right):(x, t) \in X \times T^{\prime}\right\}$

and $B\left(T^{\prime \prime}\right)=\left\{\left(x, \mu_{B}(x, t), \eta_{B}(x, t)\right):(x, t) \in X \times T^{\prime \prime}\right\}$ where $T^{\prime}$ and $T^{\prime \prime}$ have finite number of distinct time-elements or they are time intervals. Then,

$A\left(T^{\prime}\right) \cap B\left(T^{\prime \prime}\right)=\left\{\left(x, \min \left(\bar{\mu}_{A}(x, t), \bar{\mu}_{B}(x, t)\right), \max \left(\bar{\eta}_{A}(x, t), \bar{\eta}_{B}(x, t)\right):(x, t) \in X \times\left(T^{\prime} \cup T^{\prime \prime}\right)\right)\right.$ $A\left(T^{\prime}\right) \cup B\left(T^{\prime \prime}\right)=\left\{\left(x, \max \left(\bar{\mu}_{A}(x, t), \bar{\mu}_{B}(x, t)\right), \min \left(\bar{\eta}_{A}(x, t), \bar{\eta}_{B}(x, t)\right):(x, t) \in X \times\left(T^{\prime} \cup T^{\prime \prime}\right)\right)\right.$

Also from definition of subset in IFS theory, Subsets of TIFS can be defined as the follow: $A\left(T^{\prime}\right) \subseteq B\left(T^{\prime \prime}\right) \Leftrightarrow \bar{\mu}_{A}(x, t) \leq \bar{\mu}_{B}(x, t)$ and $\bar{\eta}_{A}(x, t) \geq \bar{\eta}_{B}(x, t)$ for every $(x, t) \in X \times\left(T^{\prime} \cup T^{\prime \prime}\right)$ where

$$
\begin{aligned}
& \bar{\mu}_{A}(x, t)= \begin{cases}\mu_{A}(x, t), & \text { if } t \in T^{\prime} \\
0, & \text { if } t \in T^{\prime \prime}-T^{\prime}\end{cases} \\
& \bar{\mu}_{B}(x, t)= \begin{cases}\mu_{B}(x, t) & \text { if } t \in T^{\prime \prime} \\
0, & \text { if } t \in T^{\prime}-T^{\prime \prime}\end{cases} \\
& \bar{\eta}_{A}(x, t)= \begin{cases}\eta_{A}(x, t), & \text { if } t \in T^{\prime} \\
1, & \text { if } t \in T^{\prime \prime}-T^{\prime}\end{cases} \\
& \bar{\eta}_{B}(x, t)= \begin{cases}\eta_{B}(x, t), & \text { if } t \in T^{\prime \prime} \\
1, & \text { if } t \in T^{\prime \prime}-T^{\prime}\end{cases}
\end{aligned}
$$

It is obviously seen that $\bar{\mu}_{A}(x, t)=\mu_{A}(x, t), \bar{\mu}_{B}(x, t)=\mu_{B}(x, t), \bar{\eta}_{A}(x, t)=$ $\eta_{A}(x, t), \bar{\eta}_{B}(x, t)=\eta_{B}(x, t)$ when $T^{\prime}=T^{\prime \prime}$.

Let $J$ be an arbitrary index set. Then we define that $T=\bigcup_{i \in J} T_{i}$ where $T_{i}$ is a time set for each $i \in J$. Thus, we can extend the definition of union and intersection of TIFSs family $F=\left\{A_{i}\left(T_{i}\right)=\left(x, \mu_{A_{i}}(x, t), \eta_{A_{i}}(x, t)\right): x \in X \times T_{i}, i \in J\right\}$ as follows:

$$
\bigcup_{i \in J} A\left(T_{i}\right)=\left\{\left(x, \max _{i \in J}\left(\bar{\mu}_{A_{i}}(x, t)\right), \min _{i \in J}\left(\bar{\eta}_{A_{i}}(x, t)\right):(x, t) \in X \times T\right)\right.
$$




$$
\bigcap_{i \in J} A\left(T_{i}\right)=\left\{\left(x, \min _{i \in J}\left(\bar{\mu}_{A_{i}}(x, t)\right), \max _{i \in J}\left(\bar{\eta}_{A_{i}}(x, t)\right):(x, t) \in X \times T\right),\right.
$$

where

and

$$
\bar{\mu}_{A_{j}}(x, t)= \begin{cases}\mu_{A_{j}}(x, t), & \text { if } t \in T_{j} \\ 0, & \text { if } t \in T-T_{j}\end{cases}
$$

$$
\bar{\eta}_{A_{j}}(x, t)=\left\{\begin{array}{ll}
\eta_{A_{j}}(x, t), & \text { if } t \in T_{j} \\
1, & \text { if } t \in T-T_{j}
\end{array} .\right.
$$

The operations defined above are defined over all of the time moments. In the following definition, these operations will be defined for an individual time moment.

Definition 2.8. Let

$$
\begin{aligned}
A\left(T^{\prime}\right) & =\left\{\left(x, \mu_{A}(x, t), \eta_{A}(x, t)\right):(x, t) \in X \times T^{\prime}\right\} \\
B\left(T^{\prime \prime}\right) & =\left\{\left(x, \mu_{B}(x, t), \eta_{B}(x, t)\right):(x, t) \in X \times T^{\prime \prime}\right\}
\end{aligned}
$$

where $T^{\prime}$ and $T^{\prime \prime}$ have finite number of distinct time-elements or they are time intervals. Then, the definitions of instant intersection and instant union of TIFSs are defined as follows:

$$
\begin{aligned}
A\left(T^{\prime}\right) \cap_{t_{0}} B\left(T^{\prime \prime}\right)= & \left\{\left(x, \min \left(\bar{\mu}_{A}\left(x, t_{0}\right), \bar{\mu}_{B}\left(x, t_{0}\right)\right), \max \left(\bar{\eta}_{A}\left(x, t_{0}\right), \bar{\eta}_{B}\left(x, t_{0}\right)\right):\right.\right. \\
& \left.\left(x, t_{0}\right) \in X \times\left(T^{\prime} \cup T^{\prime \prime}\right)\right\}, \\
A\left(T^{\prime}\right) \cup_{t_{0}} B\left(T^{\prime \prime}\right)= & \left\{\left(x, \max \left(\bar{\mu}_{A}\left(x, t_{0}\right), \bar{\mu}_{B}\left(x, t_{0}\right)\right), \min \left(\bar{\eta}_{A}\left(x, t_{0}\right), \bar{\eta}_{B}\left(x, t_{0}\right)\right):\right.\right. \\
& \left.\left(x, t_{0}\right) \in X \times\left(T^{\prime} \cup T^{\prime \prime}\right)\right\} .
\end{aligned}
$$

Also from definition of subset in IFS theory, instant subsets of TIFS can be defined as the following: $A\left(T^{\prime}\right) \subseteq_{t_{0}} B\left(T^{\prime \prime}\right) \Leftrightarrow \bar{\mu}_{A}\left(x, t_{0}\right) \leq \bar{\mu}_{B}\left(x, t_{0}\right)$ and $\bar{\eta}_{A}(x, t) \geq$ $\bar{\eta}_{B}(x, t)$ for every $\left(x, t_{0}\right) \in X \times\left(T^{\prime} \cup T^{\prime \prime}\right)$ where

$$
\begin{aligned}
& \bar{\mu}_{A}\left(x, t_{0}\right)= \begin{cases}\mu_{A}\left(x, t_{0}\right), & \text { if } t_{0} \in T^{\prime} \\
0, & \text { if } t_{0} \in T^{\prime \prime}-T^{\prime}\end{cases} \\
& \bar{\mu}_{B}\left(x, t_{0}\right)= \begin{cases}\mu_{B}\left(x, t_{0}\right), & \text { if } t_{0} \in T^{\prime \prime} \\
0, & \text { if } t_{0} \in T^{\prime}-T^{\prime \prime}\end{cases} \\
& \bar{\eta}_{A}\left(x, t_{0}\right)= \begin{cases}\eta_{A}\left(x, t_{0}\right), & \text { if } t_{0} \in T^{\prime} \\
1, & \text { if } t_{0} \in T^{\prime \prime}-T^{\prime}\end{cases} \\
& \bar{\eta}_{B}\left(x, t_{0}\right)= \begin{cases}\eta_{B}\left(x, t_{0}\right), & \text { if } t_{0} \in T^{\prime \prime} \\
1, & \text { if } t_{0} \in T^{\prime \prime}-T^{\prime}\end{cases}
\end{aligned}
$$

Let $J$ be an arbitrary index set. Then we define that $T=\bigcup_{i \in J} T_{i}$ where $T_{i}$ is a time set for each $i \in J$. Thus, we can extend the definition of union and intersection of TIFSs family $F_{t_{0}}=\left\{A_{i}\left(T_{i}\right)=\left(x, \mu_{A_{i}}\left(x, t_{0}\right), \eta_{A_{i}}\left(x, t_{0}\right)\right):\left(x, t_{0}\right) \in X \times T_{i}, i \in J\right\}$ as follows:

$$
\begin{aligned}
& \bigcup_{i \in J}^{t_{0}} A\left(T_{i}\right)=\left\{\left(x, \max _{i \in J}\left(\bar{\mu}_{A_{i}}\left(x, t_{0}\right)\right), \min _{i \in J}\left(\bar{\eta}_{A_{i}}\left(x, t_{0}\right)\right):\left(x, t_{0}\right) \in X \times T\right)\right. \\
& \bigcap_{i \in J}^{t_{0}} A\left(T_{i}\right)=\left\{\left(x, \min _{i \in J}\left(\bar{\mu}_{A_{i}}\left(x, t_{0}\right)\right), \max _{i \in J}\left(\bar{\eta}_{A_{i}}\left(x, t_{0}\right)\right):\left(x, t_{0}\right) \in X \times T\right)\right.
\end{aligned}
$$

where

$$
\bar{\mu}_{A_{j}}\left(x, t_{0}\right)= \begin{cases}\mu_{A_{j}}\left(x, t_{0}\right), & \text { if } t_{0} \in T_{j} \\ 0, & \text { if } t_{0} \in T-T_{j}\end{cases}
$$




$$
\bar{\eta}_{A_{j}}\left(x, t_{0}\right)=\left\{\begin{array}{ll}
\eta_{A_{j}}\left(x, t_{0}\right), & \text { if } t_{0} \in T_{j} \\
1, & \text { if } t_{0} \in T-T_{j}
\end{array} .\right.
$$

In fact, these TIFS operators can be seen as IFS operators over TIFSs, since they are defined for a single time moment.

Definition 2.9. ${\underset{\sim}{t}}^{t}$ and $\underset{\sim}{1^{t} \in T I F} S^{(X, T)}$ are defined as:

$$
0_{\sim}^{t}=\{(x, 0,1):(x, t) \in X \times T\}
$$

and

$$
\underset{\sim}{1^{t}}=\{(x, 1,0):(x, t) \in X \times T\}
$$

for each time moment t, i.e. $\mu_{0^{t}}(x, t)=0, \underset{\sim}{\eta_{0^{t}}}(x, t)=1$ and $\mu_{1^{t}}(x, t)=1$, $\eta_{1^{t}}(x, t)=0$ for each $(x, t) \in X \tilde{\times} T$.

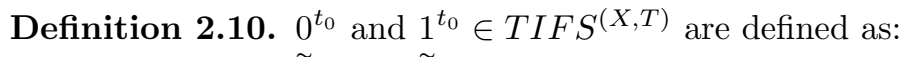

and

$$
0_{\sim}^{t_{0}}=\left\{\left(x, \underset{\sim}{\mu_{0^{t_{0}}}}(x, t), \underset{\sim}{\eta_{0^{t_{0}}}}(x, t)\right):(x, t) \in X \times T\right\}
$$

$$
{\stackrel{\sim}{1^{t}}}^{t_{0}}=\left\{\left(x, \underset{\sim}{\mu_{1^{t_{0}}}}(x, t), \eta_{\sim}^{\eta_{1}^{t_{0}}}(x, t)\right):(x, t) \in X \times T\right\}
$$

for individual time moment $t_{0} \in T$, i.e. $\mu_{0^{t}}\left(x, t_{0}\right)=0, \underset{\sim}{\eta_{0^{t}}}\left(x, t_{0}\right)=1$ and $\mu_{\sim}^{1_{0} t_{0}}\left(x, t_{0}\right)=1, \underset{\sim}{\eta_{1^{t_{0}}}}\left(x, t_{0}\right)=0$ for each $\left(x, t_{0}\right) \in X \times\left\{t_{0}\right\}$.

Definition 2.11. An temporal intuitionistic fuzzy topology in Šostak's sense (briefly, ST-TIFS) on a non-empty set $X$ is an IFF $\tau_{t}$ defined with $\tau_{t}(A)=\left(\mu_{\tau_{t}}(A), \eta_{\tau_{t}}(A)\right)$ on $X$ satisfying the following axioms for each time moment $t$

I. $\tau_{t}\left(\underset{\sim}{0^{t}}\right)=1^{\sim}$ and $\tau_{t}\left(\underset{\sim}{1^{t}}\right)=1^{\sim}$,

II. $\tau_{t}\left(A_{1} \cap A_{2}\right) \geq \tau_{t}\left(A_{1}\right) \wedge \tau_{t}\left(A_{2}\right)$ for any sets $A_{1}, A_{2} \in \operatorname{TIF} S^{(X, T)}$,

III. $\tau_{t}\left(\bigcup A_{i}\right) \geq \wedge_{i \in J}\left(\tau_{t}\left(A_{i}\right)\right)$ for $\left\{A_{i}: i \in J\right\} \subseteq T I F S^{(X, T)}$.

The pair $\left(X, \tau_{t}\right)$ is called temporal intuitionistic fuzzy topological space in Šstak sense. For any $A \in T I F S^{(X, T)}$, the number $\mu_{\tau_{t}}(A)$ is called instant openness degree of $A$ at time-moment $t$, while $\eta_{\tau_{t}}(A)$ is called instant non-openness degree of $A$ at time-moment $t$. In this definition, it is worth to note that the instant openness and the instant non-openness degree change with depending on both time and TIFS.

It is worth to note that for singleton time set $\left(X, \tau_{t}\right)$ is an intuitionistic fuzzy topology in Šostak's sense.

Proposition 1. Let $\left(X, \tau_{t}\right)$ be a ST-TIFS on $X$ and $T$ be a time-moment set. Then $\left(X, \wedge \tau_{t}\right)$ defined by $\wedge \tau_{t}(A)=\left(\min _{t \in T} \mu_{\tau_{t}}(A), \max _{t \in T} \eta_{\tau_{t}}(A)\right)$ is an intuitionistic fuzzy topology on $\operatorname{TIFS}^{(X, T)}$ in Šostak's sense.

Definition 2.12. Let $\left(X, \tau_{t}\right)$ be a ST-TIFS and $A \in T I F S^{(X, T)}$ Then we define instant closure and instant interior of $A$ at time moment $t_{0}$ according to $\tau_{t}$ respectively as:

$$
c l^{t_{0}}(A)=\bigcap\left\{K \in \operatorname{TIFS}(X) ; \tau_{t_{0}}^{*}(K)>\tilde{0}, A \subseteq K\right\}
$$


and

$$
\operatorname{int}^{t_{0}}(A)=\bigcup\left\{K \in \operatorname{TIFS}(X) ; \tau_{t_{0}}(K)>\tilde{0}, K \subseteq A\right\} .
$$

On the other hand $(\alpha, \beta)$-instant closure and $(\alpha, \beta)$-instant interior of $A$ are defined by:

$$
c l_{(\alpha, \beta)}^{t_{0}}(A)=\bigcap\left\{K \in \operatorname{TIFS}(X) ; \tau_{t_{0}}^{*}(K) \geq\langle\alpha, \beta\rangle, A \subseteq K\right\}
$$

and

$$
\operatorname{int}_{(\alpha, \beta)}^{t_{0}}(A)=\bigcup\left\{K \in \operatorname{TIFS}(X) ; \tau_{t_{0}}(K) \geq\langle\alpha, \beta\rangle, K \subseteq A\right\}
$$

where $\alpha \in(0,1], \beta \in[0,1)$ with $\alpha+\beta \leq 1$.

Definition 2.13. Let $\left(X, \tau_{t}\right)$ and $\left(Y, \phi_{t}\right)$ be ST-TIFSs respectively for non-empty sets $X, Y$, time sets $T^{\prime}$ and $T^{\prime \prime}$. Let $f: X \rightarrow Y$ be a function. Then,

(i) The preimage of $B \in \operatorname{TIFS}^{\left(Y, T^{\prime \prime}\right)}$ under $f$ at time moment $\mathrm{t}$ is defined as $f^{-1}(B)=\left\{\left(x, \bar{\mu}_{B}(f(x), t), \bar{\eta}_{B}(f(x), t)\right): x \in X\right\}$ where

$$
\begin{aligned}
& \bar{\mu}_{B}(f(x), t)= \begin{cases}\mu_{B}(f(x), t), & \text { if } t \in T^{\prime \prime} \\
0, & \text { if } t \in T^{\prime}-T^{\prime \prime}\end{cases} \\
& \bar{\eta}_{B}(f(x), t)= \begin{cases}\eta_{B}(f(x), t), & \text { if } t \in T^{\prime \prime} \\
1, & \text { if } t \in T^{\prime}-T^{\prime \prime} .\end{cases}
\end{aligned}
$$

(ii) The image of $A \in \operatorname{TIFS}\left(X, T^{\prime}\right)$ under $f$ at time moment $t$ is defined as $f(A)=\left\{\left(y, f\left(\bar{\mu}_{A}\right)(y, t), f_{-}\left(\bar{\eta}_{A}\right)(y, t)\right): y \in Y\right\}$ where

$$
\begin{gathered}
f\left(\bar{\mu}_{A}\right)(y, t)= \begin{cases}f\left(\mu_{A}\right)(y, t), & \text { if } t \in T^{\prime} \\
0, & \text { if } t \in T^{\prime \prime}-T^{\prime}\end{cases} \\
f_{-}\left(\bar{\eta}_{A}\right)(y, t)= \begin{cases}1-f\left(1-\eta_{A}\right)(y, t), & \text { if } t \in T^{\prime} \\
1, & \text { if } t \in T^{\prime \prime}-T^{\prime} .\end{cases}
\end{gathered}
$$

If $T^{\prime}=T^{\prime \prime}$, It is clearly understood that

$$
f^{-1}(B)=\left\{\left(x, \mu_{B}(f(x), t), \eta_{B}(f(x), t)\right): x \in X\right\}
$$

and

$$
f(A)=\left\{\left(y, f\left(\mu_{A}\right)(y, t), f_{-}\left(\eta_{A}\right)(y, t)\right): y \in Y\right\} .
$$

Let $\left(X, \tau_{t}\right)$ and $\left(Y, \phi_{t}\right)$ be ST-TIFSs for non-empty sets $X, Y$ and time set $T$. If $\tau_{t}\left(f^{-1}(B)\right) \geq \phi_{t}(B)$ for $t \in T$ and each $B \in T I F S^{(Y, T)}, f$ is called temporal intuitionistic fuzzy continuous function at time moment $t$. If $f$ is temporal intuitionistic fuzzy continuous function at each time moment, $f$ is called overall intuitionistic fuzzy continuous function.

On the other hand, If $\phi_{t}(f(A)) \geq \tau_{t}(A)$ for $t \in T$ and each $A \in T I F S^{\left(X, T^{\prime}\right)}$, $f$ is called temporal intuitionistic fuzzy open function at time moment $t$. If $f$ is temporal intuitionistic fuzzy open function at each time moment, $f$ is called overall intuitionistic fuzzy open function. 


\section{MAIN RESULTS}

In this section, we will first give definition of temporal intuitionistic fuzzy topology in Chang's sense and other some basic definitions related this concept. We will then generalize these concepts under the name of the overall intuitionistic fuzzy topology in Chang's sense.

Definition 3.1. An temporal intuitionistic fuzzy topology in Chang's sense (briefly, CT-TIFS) on a non-empty set $X$ is a family $\tau_{t_{0}}$ of TIFSs satisfying the following axioms for fixed time moment $t_{0}$

I. $0^{t_{0}} \in \tau_{t_{0}}$ and $1^{t_{0}} \in \tau_{t_{0}}$,

II. For each $A_{1}, \tilde{A}_{2} \in \tau_{t_{0}}$, there exist a $F \in \tau_{t_{0}}$ such that $\mu_{F}\left(x, t_{0}\right)=\mu_{A_{1} \cap_{t_{0}} A_{2}}\left(x, t_{0}\right)$, $\eta_{F}\left(x, t_{0}\right)=\eta_{A_{1} \cap_{t_{0}} A_{2}}\left(x, t_{0}\right)$ for each $\left(x, t_{0}\right) \in X \times\left\{t_{0}\right\}$.

III. For any arbitrary family $\left\{A_{i} ; i \in I\right\} \in \tau_{t_{0}}$, there exist a $D \in \tau_{t_{0}}$ such that $\mu_{D}\left(x, t_{0}\right)=\mu_{i \in I}^{t_{0} A_{i}}\left(x, t_{0}\right)$ and $\eta_{D}\left(x, t_{0}\right)=\eta_{i \in I}^{\cup_{0}{ }_{0} A_{i}}\left(x, t_{0}\right)$ for each $\left(x, t_{0}\right) \in X \times$ $\left\{t_{0}\right\}$.

The pair $\left((X, T), \tau_{t_{0}}\right)$ is called temporal intuitionistic fuzzy topological space in Chang's sense. Any member of $\tau_{t_{0}}$ is called temporal intuitionistic fuzzy open set (TIFOS). On the other hand, the complement of any member of $\tau_{t_{0}}$ is called intuitionistic fuzzy closed set (TIFCS). It is obtained intuitionistic fuzzy topological space in Chang's sense from every temporal intuitionistic fuzzy topological space in Chang's sense by the following method.

Proposition 2. Let $\tau_{t_{0}}$ is an temporal intuitionistic fuzzy topological space in Chang's sense on non-empty set $X$ and time moment set $T$, Then we define IFS's from every $A \in \tau_{t_{0}}$ TIFSs by following way: $\mu_{\hat{A}}(x)=\mu_{A_{i}}\left(x, t_{0}\right)$ and $\eta_{\hat{A}}(x)=$ $\eta_{A}\left(x, t_{0}\right)$. So that the new family $\tau^{t_{0}}=\left\{\hat{A}: A \in \tau_{t_{0}}\right\}$ obtained from $\tau_{t_{0}}$ is a intuitionistic fuzzy topology in Chang's sense.

Lowen stated in [11] that the constant functions in the topology defined by the Chang approach are not continuous. Therefore, Lowen corrected this deficiency by changing the first axiom in Chang's definition. In the literature these two definitions are treated as equivalent.

Definition 3.2. An temporal intuitionistic fuzzy topology in Lowen's sense (briefly, LT-TIFS) on a non-empty set $X$ is a family $\tau_{t_{0}}$ of TIFSs satisfying the following axioms for fixed time moment $t_{0}$

I. For any fixed IF-pair $(\alpha, \beta)$, let define TIFSs

$$
A=\left\{\left(x, \mu_{A}(x, t), \eta_{A}(x, t)\right):(x, t) \in X \times T\right\}
$$

such that $\mu_{A}\left(x, t_{0}\right)=\alpha, \eta_{A}\left(x, t_{0}\right)=\beta$ for every $\left(x, t_{0}\right) \in X \times\left\{t_{0}\right\}$.

II. For each $A_{1}, A_{2} \in \tau_{t_{0}}$, there exist a $F \in \tau_{t_{0}}$ such that $\mu_{F}\left(x, t_{0}\right)=\mu_{A_{1} \cap_{t_{0}} A_{2}}\left(x, t_{0}\right)$, $\eta_{F}\left(x, t_{0}\right)=\eta_{A_{1} \cap_{t_{0}} A_{2}}\left(x, t_{0}\right)$ for each $\left(x, t_{0}\right) \in X \times\left\{t_{0}\right\}$.

III. For any arbitrary family $\left\{A_{i} ; i \in I\right\} \in \tau_{t_{0}}$, there exist a $D \in \tau_{t_{0}}$ such that $\mu_{D}\left(x, t_{0}\right)=\mu \bigcup_{i \in I}^{t_{0} A_{i}}\left(x, t_{0}\right), \eta_{D}\left(x, t_{0}\right)=\eta_{i \in I}{ }_{i \in I}^{t_{0}} A_{i}\left(x, t_{0}\right)$ for each $\left(x, t_{0}\right) \in X \times\left\{t_{0}\right\}$. We will use our definition of the temporal intuitionistic fuzzy topology in Chang's sense topology in the rest of the work. 
Definition 3.3. Let $\tau_{t_{0}}$ is an temporal intuitionistic fuzzy topological space in Chang's sense on non-empty set $X$ and time moment set $T$ and $A \in \tau_{t_{0}}$. Then temporal intuitionistic fuzzy interior and temporal intuitionistic fuzzy closure of $A$ defined as follows: $\operatorname{int}_{t_{0}}(A)=\cup\left\{G ; G \in \tau_{t_{0}}, G \subseteq A\right\}, c l_{t_{0}}(A)=\cap\left\{C ; \bar{C} \in \tau_{t_{0}}, A \subseteq C\right\}$. Following propositions are valid for both of fuzzy and intuitionistic fuzzy case $[1,2,3,4,10,11,12]$, it can be proved as in the above-mentioned articles.

Definition 3.4. Let $\tau_{t_{0}}$ be a temporal intuitionistic fuzzy topological space in Chang's sense on non-empty set $X$ and time moment set. Then,

i. $A$ is a TIFCS in $\tau_{t_{0}} \Leftrightarrow c l_{t_{0}}(A)=A$,

ii. $A$ is a TIFOS in $\tau_{t_{0}} \Leftrightarrow \operatorname{int}_{t_{0}}(A)=A$,

iii. $c l_{t_{0}}(A)=\overline{\operatorname{int}_{t_{0}}(A)}$ for any $A \in T I F S^{(X, T)}$,

iv. $\operatorname{int}_{t_{0}}(A)=\overline{c l_{t_{0}}(A)}$ for any $A \in T I F S^{(X, T)}$,

v. $\operatorname{int}_{t_{0}}(A) \subseteq A$ for any $A \in T I F S^{(X, T)}$,

vi. $A \subseteq c l_{t_{0}}(A)$ for any $A \in T I F S^{(X, T)}$,

vii. $A \subseteq B \Rightarrow \operatorname{int}_{t_{0}}(A) \subseteq \operatorname{int}_{t_{0}}(B)$ for any $A, B \in T I F S^{(X, T)}$,

viii. $A \subseteq B \Rightarrow c l_{t_{0}}(A) \subseteq c l_{t_{0}}(B)$ for any $A, B \in T I F S^{(X, T)}$,

ix. $c l_{t_{0}}\left(c l_{t_{0}}(A)\right)=c l_{t_{0}}(A)$ for any $A \in T I F S^{(X, T)}$,

x. $\operatorname{int}_{t_{0}}\left(\operatorname{int}_{t_{0}}(A)\right)=\operatorname{int}_{t_{0}}(A)$ for any $A \in T I F S^{(X, T)}$,

xi. $\operatorname{int}_{t_{0}}(A \cap B)=\operatorname{int}_{t_{0}}(A) \cap \operatorname{int}_{t_{0}}(B)$ for any $A, B \in T I F S^{(X, T)}$,

xii. $c l_{t_{0}}(A \cup B)=c l_{t_{0}}(A) \cup c l_{t_{0}}(B)$ for any $A, B \in T I F S^{(X, T)}$,

xiii. $\operatorname{int}_{t_{0}}\left(\underset{\sim}{1^{t_{0}}}\right)={\underset{\sim}{1}}^{t_{0}}$

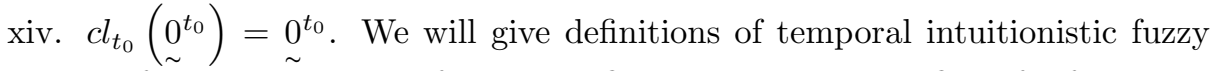
continuous functions and open function definitions, which are defined for fuzzy and intuitionistic fuzzy sets in $[1,2,3,4,10,11,12]$, respectively.

Definition 3.5. Let $\left(\left(X, T^{\prime}\right), \tau_{t_{0}}\right)$ and $\left(\left(Y, T^{\prime \prime}\right), \phi_{t_{0}}\right)$ be two CT-TIFSs and let $f: X \rightarrow Y$ be a function. Then $f$ said to be temporal intuitionistic fuzzy continuous at time moment $t_{0}$ if and only if the temporal preimage of each TIFS in $\phi_{t_{0}}$ is an TIFS in $\tau_{t_{0}}$

Definition 3.6. Let $\left(\left(X, T^{\prime}\right), \tau_{t_{0}}\right)$ and $\left(\left(Y, T^{\prime \prime}\right), \phi_{t_{0}}\right)$ be two CT-TIFSs and let $f: X \rightarrow Y$ be a function. Then $f$ said to be temporal intuitionistic fuzzy open at time moment $t_{0}$ if and only if the temporal image of each TIFS in $\tau_{t_{0}}$ is an TIFS in $\phi_{t_{0}}$.

The following propositions are valid for fuzzy and intuitionistic fuzzy topological spaces in Chang's sense (it can be seen from $[2,3,4,10,12]$ ) and these propositions are generalized for temporal intuitionistic fuzzy topological spaces in Chang's sense as follows.

Proposition 3. $f:\left(\left(X, T^{\prime}\right), \tau_{t_{0}}\right) \rightarrow\left(\left(Y, T^{\prime \prime}\right), \phi_{t_{0}}\right)$ is temporal intuitionistic fuzzy continuous iff the temporal preimage of each TIFCS in $\phi_{t_{0}}$ is an TIFCS in $\tau_{t_{0}}$.

Proposition 4. The following statements are equivalent to each other a. $f$ : $\left(\left(X, T^{\prime}\right), \tau_{t_{0}}\right) \rightarrow\left(\left(Y, T^{\prime \prime}\right), \phi_{t_{0}}\right)$ is temporal intuitionistic fuzzy continuous, b. $f^{-1}\left(\right.$ int $\left._{t_{0}}(B)\right) \subseteq$ $\operatorname{int}_{t_{0}} f^{-1}(B)$ for each $B \in T I F S^{\left(Y, T^{\prime \prime}\right)}$, c. $c l_{t_{0}}\left(f^{-1}(B)\right) \subseteq f^{-1}\left(c l_{t_{0}}(B)\right)$ for each $B \in \operatorname{TIFS}^{\left(Y, T^{\prime \prime}\right)}$.

The relation, which is previously described by Šstak [1], Mondal and Samanta [10] for fuzzy and intuitionistic fuzzy sets, between temporal intuitionistic fuzzy 
topologies Chang and Šostak's senses will be expanded. The proof of this proposition is not given because it is quite similar in the above-mentioned articles $[1,10]$.

Proposition 5. Let $\tau_{t_{0}}$ be an temporal intuitionistic fuzzy topological space in Chang's sense on non-empty set $X$ and time moment set and $\left\langle\alpha_{t_{0}}, \beta_{t_{0}}\right\rangle$ is an IFpair. Then, the IFF $\tau_{t_{0}}^{\left\langle\alpha_{t_{0}}, \beta_{t_{0}}\right\rangle}$ which is defined as follows:

$$
\tau_{t_{0}}^{\left\langle\alpha_{t_{0}}, \beta_{t_{0}}\right\rangle}(A)= \begin{cases}\left\langle\alpha_{t_{0}}, \beta_{t_{0}}\right\rangle, & \text { if } A \in \tau_{t_{0}}-\left\{\underset{\sim}{0^{t}},{\underset{\sim}{t}}^{t}\right\} \\ \langle 1,0\rangle, & \text { if } A \in\left\{\underset{\sim}{0^{t}, \sim_{\sim}^{t}}\right\}_{\sim} \\ \langle 0,1\rangle, & \text { if } A \notin \tau_{t_{0} 0}-\left\{\underset{\sim}{0^{t}, \sim_{\sim}^{t}}\right\}\end{cases}
$$

is a temporal intuitionistic fuzzy topology in Šostak's sense. This ST-TIFS is called as $\left\langle\alpha_{t_{0}}, \beta_{t_{0}}\right\rangle$ - ST-TIFS .

On the other hand, Let $\tau_{t_{0}}^{*}$ is an temporal intuitionistic fuzzy topological space in Šostak's sense on non-empty set $X$ and time moment set and $\left\langle\alpha_{t_{0}}, \beta_{t_{0}}\right\rangle$ is an IF-pair. Then, the family of TIFSs $\tau_{t_{0}}^{*\left\langle\alpha_{t_{0}}, \beta_{t_{0}}\right\rangle}$ which is defined as follows:

$A \in \tau_{t_{0}}^{*\left\langle\alpha_{t_{0}}, \beta_{t_{0}}\right\rangle} \Leftrightarrow \tau_{t_{0}}^{*}(A) \geq\left\langle\alpha_{t_{0}}, \beta_{t_{0}}\right\rangle$ is a temporal intuitionistic fuzzy topology in Šostak's sense.

As mentioned in the article of Mondal and Samanta [10], between these two topologies, being the continuous function (or open function) is interrelated. In other words, a function that is continuous (open) function in one topological space is continuous (open) in the other topological space. Moreover, the another concept it will be called the overall intuitionistic fuzzy topology in Chang's sense, which will be valid for the entire set of time we are dealing with, is defined as follows.

Definition 3.7. An overall intuitionistic fuzzy topology in Chang's sense (briefly, OCT-TIFS) on a non-empty set $X$ is a family $\tau_{t}$ of TIFSs satisfying the following axioms for each time moment $t$ I. $0^{t} \in \tau_{t}$ and $1^{t} \in \tau_{t}$, II. For each $A_{1}, A_{2} \in \tau_{t}$, there exist a $F \in \tau_{t}$ such that $\mu_{F}(x, t) \stackrel{\sim}{=} \mu_{A_{1} \cap A_{2}}(\tilde{x}, t), \eta_{F}(x, t)=\eta_{A_{1} \cap_{t} A_{2}}(x, t)$ for each $(x, t) \in X \times T$. III. For any arbitrary family $\left\{A_{i} ; i \in I\right\} \in \tau_{t}$, there exist a $D \in \tau_{t}$ such that $\mu_{D}(x, t)=\mu_{i \in I} A_{i}(x, t), \eta_{D}(x, t)=\eta_{i \in I} A_{i}(x, t)$ for each $(x, t) \in X \times T$.

By this definition, it is understood that a temporal intuitionistic fuzzy topology in Chang's sense can be obtained for each time moment in an overall intuitionistic fuzzy topology in Chang's sense.

Theorem 3.8. Let $\tau_{t}$ be a overall intuitionistic fuzzy topological space in Chang's sense on non-empty set $X$ and finite time moment set T. Then, family of TIFSs $\tau$ which defined as: $\underset{A \in \tau_{t}}{\cap} A=\left\{\left\langle x, \underset{t \in T}{\wedge} \mu_{A}(x, t), \underset{t \in T}{\vee} \eta_{A}(x, t)\right\rangle\right\} \in \tau$ is a CT-IFS on TIFS.

Proof. In order to prove the theorem, it is necessary to show that the conditions given in the definition 2.5 .

i. Since $\underset{\sim}{0^{t_{0}}} \in \tau_{t}$ for each $t_{0} \in T, \underset{t_{0} \in T}{\wedge} 0_{\sim}^{t_{0}}(x, t)=0$ and $\underset{t_{0} \in T}{\vee} \stackrel{0}{\sim}^{t_{0}}(x, t)=1$, then $\underset{t_{0} \in T \sim}{\cap} 0^{t_{0}}=0_{\sim}^{t}$. Then it is understood that $0_{\sim}^{t} \in \tau$. It can be shown by similar method 


$$
\begin{aligned}
& 0_{\sim}^{t} \in \tau \text {. ii. Let } \widehat{A_{i}}=\underset{A_{i} \in \tau_{t}}{\cap} A_{i}=\left\{\left\langle x, \underset{t \in T}{\wedge} \mu_{A_{i}}(x, t), \underset{t \in T}{\vee} \eta_{A_{i}}(x, t)\right\rangle\right\} \text { and } \widehat{A_{j}}= \\
& \cap_{A_{j} \in \tau_{t}} A_{j}=\left\{\left\langle x, \underset{t \in T}{\wedge} \mu_{A_{j}}(x, t), \underset{t \in T}{\vee} \eta_{A_{j}}(x, t)\right\rangle\right\} \in \tau \text {. Then } \widehat{A_{i}} \cap \widehat{A_{j}}=\left(\underset{A_{i} \in \tau_{t}}{\cap} A_{i}\right) \cap \\
& \left(\cap_{A_{j} \in \tau_{t}} A_{j}\right)=\left\{\left\langle x,\left(\hat{t \in T}_{t} \mu_{A i}(x, t)\right) \wedge\left(\hat{\wedge}_{t \in T} \mu_{A_{j}}(x, t)\right),\left(\underset{t \in T}{\vee} \eta_{A i}(x, t)\right) \vee\left(\vee_{t \in T} \eta_{A_{j}}(x, t)\right)\right\rangle\right\} \\
& =\left\{\left\langle x,\left(\underset{t \in T}{\wedge}\left(\mu_{A i}(x, t) \wedge \mu_{A_{j}}(x, t)\right)\right),\left(\underset{t \in T}{\vee} \eta_{A i}(x, t) \vee \eta_{A_{j}}(x, t)\right)\right\rangle\right\}=\bigcap_{t \in T} A_{i} \cap A_{j} .
\end{aligned}
$$

Since $\tau_{t}$ is OCT-TIFS, it is understood that $\widehat{A_{i}} \cap \widehat{A_{j}} \in \tau$

iii. It can be proved similar method ii

\section{Conclusion}

In this paper, the concepts of temporal and overall intuitionistic fuzzy topology in Chang sense is introduced. On the other hand, the concept of fuzzy topology defined by Lowen was transferred to this new space. These new definitions have been found to provide basic relationships in fuzzy and intuitionistic fuzzy topologies in general. Also at the end of the study, a way to obtain overall intuitionistic fuzzy topology from intuitionistic fuzzy topology is presented. This shows that the topologies we define are more comprehensive than other topologies.

\section{REFERENCES}

[1] A.Šostak, On a fuzzy topological structure. Rend Circ. Mat. Palermo Supp., Vol. 11,pp. 89-103 (1985).

[2] C. L. Chang, Fuzzy topological spaces. J. Math Ana. Appl.,Vol. 24, 182-190.(1968)

[3] D. Çoker and M. Demirci, An introduction to intuitionistic topological spaces in Šostak's sense. BUSEFAL, Vol. 67,pp. 67-76 (1996).

[4] D. Çoker, An introduction to intuitionistic fuzzy topological spaces. Fuzzy sets and systems Vol. 88, N. 1, pp. 81-89 (1997).

[5] F.Kutlu, O. Atan and T. Bilgin, Distance measure, similarity measure, entropy and inclusion measure for temporal intuitionistic fuzzy sets. In: Proceedings of IFSCOM'2016, Mersin/Turkey, pp.130-148 (2016).

[6] F. Kutlu ,T. Bilgin , Temporal intuitionistic fuzzy topology in Šostak's sense. Notes on Intuitionistic Fuzzy Sets, Vol. 21 N.2, pp. 63-70, (2015).

[7] F.Kutlu , A. A. Ramadan ,T. Bilgin, On compactness in temporal intuitionistic fuzzy Šostak topology, Notes on Intuitionistic Fuzzy Sets, Vol 22, N. 5, pp. 46-62.(2016).

[8] K. T. Atanassov, Intuitionistic fuzzy sets. Fuzzy Sets and Systems, Vol. 20, N.1, pp. 87-96 (1986).

[9] K. T. Atanassov, Temporal intuitionistic fuzzy sets. Comptes Rendus de l'Academie Bulgare,Vol. 44, N.7,pp. 5-7 (1991).

[10] S. K. Samanta , T. K.Mondal, Intuitionistic gradation of openness: intuitionistic fuzzy topology. BUSEFAL, Vol. 73,pp. 8-17 (1997).

[11] R. Lowen, Fuzzy topological spaces and fuzzy compactness. Journal of Mathematical Analysis and Applications, Vol. 56, N.3,pp. 621-633 (1976).

[12] S. J. Lee, E. P. Lee, The category of intuitionistic fuzzy topological spaces. Bulletin of the Korean Mathematical Society, Vol 37, N.1,pp. 63-76 (2000).

[13] S. Yılmaz, G. Çuvalcıŏlu, On level operators for temporal intuitionistic fuzzy sets. Notes on Intuitionistic Fuzzy Sets, Vol. 20, N.2,pp. 6-15 (2014).

Van YÜzüncü Yil University, Mathematics Department, 65080, Van, TURKEY

Email address: fatihkutlu@yyu.edu.tr 